\title{
A reduction technique in Waring's problem, I
}

by

\author{
Kent D. Boklan (Nashville, Tenn.)
}

1. Introduction. Often, when considering the integral over the minor arcs in the Hardy-Littlewood circle method, one resorts to bounding the number of solutions of an underlying diophantine equation. If such a calculation is not contingent upon the structure of the underlying sets to which the variables are restricted, one may save a power of a logarithm in the considered error term by thinning the sets over which the variables lie to ones of smaller cardinality. The main result of this paper is the following theorem concerning the minor arc estimate associated with the representation of an integer by eight cubes from (ostensibly) arbitrary sets.

Theorem. Suppose that $\mathfrak{m} \subseteq[0,1]$,

$$
G_{i}(\alpha)=\sum_{n \in \mathcal{G}_{i}} e\left(\alpha n^{3}\right), \quad \mathcal{G}_{i} \subseteq[1, P] \cap \mathbb{Z},
$$

and that, for some $\gamma$,

$$
\sup _{\alpha \in \mathfrak{m}}\left|G_{1}(\alpha)\right| \ll P^{3 / 4}(\log P)^{\gamma} .
$$

Then, for any $\varepsilon>0$,

$$
\int_{\mathfrak{m}} \prod_{i=1}^{8}\left|G_{i}(\alpha)\right| d \alpha \ll P^{5}(\log P)^{\varepsilon-3} .
$$

This result is achieved through the use of a rather general "reduction" lemma (Lemma D). It may well be the case that lemma will prove of greater interest than the above theorem. For practical purposes, restrictions need be placed on such sets $\mathcal{G}_{i}$. They should possess suitably reasonable distribution properties (e.g. analogues of the Siegel-Walfisz Theorem) so that the major arcs can be readily handled (the generating functions $G_{i}(\alpha)$ may be well-approximated) to obtain asymptotic formulae. Additionally, the $\mathcal{G}_{i}$ should be sufficiently dense (else the minor arc bound as in the Theorem will overwhelm the major arc contribution). Of course a Weyl-type minor arc estimate superior to the one given above would lead to much more satisfactory 
conclusions. The argument presented herein is limited to the case of eight cubes, but a much more general result may be constructed. We intend to provide such a method in a sequel paper. The techniques that are involved for higher powers are quite different and it is therefore more appropriate to consider them separately.

We provide two applications of our technique - first improving upon the error term as deduced by Vaughan [6] in his important paper that established the asymptotic formula for eight cubes.

COROLlaRY 1. The number of representations, $r_{8,3}(n)$, of $n$ as the sum of eight cubes of positive integers satisfies, for every $\varepsilon>0$,

$$
r_{8,3}(n)=\mathfrak{S}_{8,3}(n) \frac{\Gamma(4 / 3)^{8}}{\Gamma(8 / 3)} n^{5 / 3}+O\left(n^{5 / 3}(\log n)^{\varepsilon-3}\right) .
$$

Here $\mathfrak{S}_{8,3}(n)$ is the usual singular series satisfying $1 \gg \mathfrak{S}_{8,3}(n) \gg 1$ (see, for example, [5, Theorem 4.6]). Vaughan [6] proved the asymptotic formula with an error of order $(\log n)^{4 / \pi-2+\varepsilon}$ smaller than the main term. The power of the logarithm in his result may be improved to $\varepsilon-1$ by employing the sharp results of Hall and Tenenbaum for Hooley's $\Delta$ function (see [2]).

Roth [4] has proved that all large integers are the sum of a cube and seven cubes of primes. He does not, however, establish any asymptotic formulae. Our methods do provide asymptotic results, but for a fewer number of prime cubic summands.

COROLlaRY 2. The number of representations, $R_{6,2}(n)$, of $n$ as the sum of six cubes of positive integers and two cubes of primes satisfies

$$
R_{6,2}(n)=\mathfrak{S}(n) C n^{5 / 3}(\log n)^{-2}+O\left(n^{5 / 3}(\log n)^{\varepsilon-3}\right)
$$

where $C$ is an absolute positive constant,

$$
\mathfrak{S}(n)=\sum_{q=1}^{\infty} \sum_{\substack{a=1 \\(a, q)=1}}^{q} q^{-6} \phi(q)^{-2} S(q, a)^{6} S^{*}(q, a)^{2} e\left(-\frac{a n}{q}\right),
$$

$\phi(q)$ is Euler's totient function, and

$$
S(q, a)=\sum_{r=1}^{q} e\left(\frac{a r^{3}}{q}\right), \quad S^{*}(q, a)=\sum_{\substack{r=1 \\(r, q)=1}}^{q} e\left(\frac{a r^{3}}{q}\right) .
$$

The proof of Lemma D is based upon a lecture of R. C. Vaughan given at the University of Michigan in 1989. Lemma D, in its fundamental form, is an iterative base for the technique introduced by Vaughan [6] to obtain the asymptotic formula for eight cubes and exploited more fully in [7] to discern the veracity of the asymptotic formula for $2^{k} k$ th powers when $k>3$. 
The author would like to thank Professors H. L. Montgomery, R. C. Vaughan, and T. D. Wooley for their support and many helpful conversations. This memoir comprises a part of the author's Ph.D. thesis at the University of Michigan.

2. Notation. As usual, $\varepsilon$ denotes a sufficiently small positive number (which may change from one occurrence to the next), $\ll$ and $\gg$ denote Vinogradov's well-known notation (where implicit constants are functions of, at most, $\varepsilon$ and $\varrho$ unless otherwise specified), and $e(x)=\exp (2 \pi i x)$. We shall also assume that $n$ is large (in terms of $\varepsilon$ ) and that

$$
F(\alpha)=\sum_{n=1}^{P} e\left(\alpha n^{3}\right),
$$

the standard generating function for cubes, and that

$$
G_{i}(\alpha)=\sum_{n \in \mathcal{G}_{i}} e\left(\alpha n^{3}\right), \quad \mathcal{G}_{i} \subseteq[1, P] \cap \mathbb{Z} .
$$

3. A reduction lemma. For primes $p$ and for fixed $\varrho$ let

$$
\mathcal{X}=\mathcal{X}(\varrho)=\left\{1 \leq n \leq P: p \mid n \text { implies } p \notin\left((\log P)^{\varrho}, P^{1 / 7}\right]\right\} .
$$

We presently record several lemmata for future reference.

Lemma A (Hooley, [3, Theorem 2]). We have

$$
\int_{0}^{1}|F(\alpha)|^{4} d \alpha \asymp P^{2} \text {. }
$$

Lemma B (Vaughan, [6, Theorem 2]). We have

$$
\int_{0}^{1}|F(\alpha)|^{8} d \alpha \asymp P^{5} .
$$

Lemma C (Vaughan, [6, Lemma 5]). Let E denote the number of solutions of

$$
x_{1}^{3}+m^{3}\left(y_{1}^{3}+y_{2}^{3}\right)=x_{2}^{3}+m^{3}\left(y_{3}^{3}+y_{4}^{3}\right)
$$

where $1 \leq x_{i} \leq P, 1 \leq y_{i} \leq P / M, M<m \leq 2 M,\left(x_{i}, m\right)=1$, and $M \leq P^{1 / 7}$. Then

$$
E \ll P^{7 / 2} M^{-9 / 2}(\log P)^{5} .
$$

We now present the main result of this section.

Lemma D. Suppose that $\mathfrak{m} \subseteq[0,1]$ and that, for some $\gamma>0$,

$$
\sup _{\alpha \in \mathfrak{m}}\left|G_{3}(\alpha)\right| \ll P^{3 / 4}(\log P)^{\gamma} .
$$


If $\varrho$ is sufficiently large, for any fixed $\delta>0$,

$$
\begin{aligned}
\int_{\mathfrak{m}} & \prod_{i=1}^{8}\left|G_{i}(\alpha)\right| d \alpha \\
& \ll \prod_{i=1}^{2}\left(\int_{\mathfrak{m}}\left|\sum_{n \in \mathcal{X}(\varrho) \cap \mathcal{G}_{i}} e\left(\alpha n^{3}\right)\right|^{2} \prod_{j=3}^{8}\left|G_{j}(\alpha)\right| d \alpha\right)^{1 / 2}+P^{5}(\log P)^{-\delta} .
\end{aligned}
$$

Pro of. Let $\mathcal{B}_{i}$ be subsets of $[1, P]$ for $i=1,2, \ldots, 8$ and set

$$
H_{i}(\alpha)=\sum_{n \in \mathcal{B}_{i}} e\left(\alpha n^{3}\right) .
$$

By Hölder's inequality,

$$
\int_{\mathfrak{m}} \prod_{i=1}^{8}\left|H_{i}(\alpha)\right| d \alpha \leq \prod_{i=1}^{8}\left(\int_{\mathfrak{m}}\left|H_{i}(\alpha)\right|^{8} d \alpha\right)^{1 / 8} .
$$

Extending the range of integration and considering the underlying diophantine equations,

$$
\int_{\mathfrak{m}}\left|H_{i}(\alpha)\right|^{8} d \alpha \leq \int_{0}^{1}\left|H_{i}(\alpha)\right|^{8} d \alpha \leq \int_{0}^{1}|F(\alpha)|^{8} d \alpha
$$

so, by Lemma B,

$$
\int_{\mathfrak{m}} \prod_{i=1}^{8}\left|H_{i}(\alpha)\right| d \alpha \ll P^{5} .
$$

By the Cauchy-Schwarz inequality,

$$
\int_{\mathfrak{m}} \prod_{i=1}^{8}\left|G_{i}(\alpha)\right| d \alpha \leq \prod_{i=1}^{2}\left(\int_{\mathfrak{m}}\left|G_{i}(\alpha)\right|^{2} \prod_{j=3}^{8}\left|G_{j}(\alpha)\right| d \alpha\right)^{1 / 2}
$$

so, by (3.1), it suffices to establish that for $i=1$ and $i=2$,

$$
\begin{aligned}
\int_{\mathfrak{m}}\left|G_{i}(\alpha)\right|^{2} \prod_{j=3}^{8}\left|G_{j}(\alpha)\right| d \alpha & \\
& \ll \int_{\mathfrak{m}}\left|\widehat{G}_{i}(\alpha)\right|^{2} \prod_{j=3}^{8}\left|G_{j}(\alpha)\right| d \alpha+P^{5}(\log P)^{-2 \delta}
\end{aligned}
$$

where we define

$$
\widehat{G}_{i}(\alpha)=\sum_{n \in \mathcal{X} \cap \mathcal{G}_{i}} e\left(\alpha n^{3}\right) .
$$


Without loss of generality we fix $i$ to be 1 . Set

$$
\begin{aligned}
\mathfrak{A} & =\left\{(x, y) \in \mathcal{G}_{1} \times \mathcal{G}_{1}:(x, y)>(\log P)^{\varrho}\right\}, \\
\mathfrak{B} & =\left\{(x, y) \in \mathcal{G}_{1} \times \mathcal{G}_{1}:(x, y) \leq(\log P)^{\varrho}, x \notin \mathcal{X}\right\}, \\
\mathfrak{C} & =\left\{(x, y) \in \mathcal{G}_{1} \times \mathcal{G}_{1}:(x, y) \leq(\log P)^{\varrho}, x \in \mathcal{X}, y \notin \mathcal{X}\right\}, \\
\mathfrak{D} & =\left\{(x, y) \in \mathcal{G}_{1} \times \mathcal{G}_{1}:(x, y) \leq(\log P)^{\varrho}, x \in \mathcal{X}, y \in \mathcal{X}\right\} .
\end{aligned}
$$

Clearly $\mathfrak{A}, \mathfrak{B}, \mathfrak{C}$, and $\mathfrak{D}$ partition $\mathcal{G}_{1} \times \mathcal{G}_{1}$ so that

$$
\begin{aligned}
\left|G_{1}(\alpha)\right|^{2} & =\sum_{x, y \in \mathcal{G}_{1}} e\left(\alpha\left(x^{3}-y^{3}\right)\right) \\
& =\left(\sum_{(x, y) \in \mathfrak{A}}+\sum_{(x, y) \in \mathfrak{B}}+\sum_{(x, y) \in \mathfrak{C}}+\sum_{(x, y) \in \mathfrak{D}}\right) e\left(\alpha\left(x^{3}-y^{3}\right)\right) .
\end{aligned}
$$

We now define, for any subset $\mathcal{S}$ of $\mathcal{G}_{1} \times \mathcal{G}_{1}$,

$$
\mathcal{J}(\mathcal{S})=\int_{\mathfrak{m}} \sum_{(x, y) \in \mathcal{S}} e\left(\alpha\left(x^{3}-y^{3}\right)\right) \prod_{j=3}^{8}\left|G_{j}(\alpha)\right| d \alpha .
$$

The integral on the left-hand side of (3.2) (for $i=1$ ) is thus equal to $\mathcal{J}(\mathfrak{A})+\mathcal{J}(\mathfrak{B})+\mathcal{J}(\mathfrak{C})+\mathcal{J}(\mathfrak{D})$.

We first consider $\mathcal{J}(\mathfrak{A})$. By the triangle inequality,

$$
\mathcal{J}(\mathfrak{A}) \leq \sum_{d>(\log P)^{e}} \int_{\mathfrak{m}}\left|\sum_{\substack{(d x, d y) \in \mathfrak{A} \\(x, y)=1}} e\left(\alpha d^{3}\left(x^{3}-y^{3}\right)\right)\right| \prod_{j=3}^{8}\left|G_{j}(\alpha)\right| d \alpha
$$

and, by Hölder's inequality, this is at most

$$
\sum_{d>(\log P)^{\varrho}}\left(\int_{\mathfrak{m}}\left|\sum_{\substack{(d x, d y) \in \mathfrak{A} \\(x, y)=1}} e\left(\alpha d^{3}\left(x^{3}-y^{3}\right)\right)\right|^{4} d \alpha\right)^{1 / 4} \prod_{j=3}^{8}\left(\int_{\mathfrak{m}}\left|G_{j}(\alpha)\right|^{8} d \alpha\right)^{1 / 8} .
$$

Using (3.1) and extending the range of integration we find that

$$
\mathcal{J}(\mathfrak{A}) \ll P^{15 / 4} \sum_{d>(\log P)^{\varrho}}\left(\int_{0}^{1}\left|\sum_{\substack{(d x, d y) \in \mathfrak{A} \\(x, y)=1}} e\left(\alpha d^{3}\left(x^{3}-y^{3}\right)\right)\right|^{4} d \alpha\right)^{1 / 4} .
$$

Bounding the number of solutions of the underlying diophantine equation, we see that

is at most

$$
\int_{0}^{1}\left|\sum_{\substack{(d x, d y) \in \mathfrak{A} \\(x, y)=1}} e\left(\alpha d^{3}\left(x^{3}-y^{3}\right)\right)\right|^{4} d \alpha
$$

$$
\#\left\{x_{1}^{3}-y_{1}^{3}+x_{2}^{3}-y_{2}^{3}=x_{3}^{3}-y_{3}^{3}+x_{4}^{3}-y_{4}^{3}: x_{i} \leq P / d, y_{i} \leq P / d\right\}
$$


which is equal to

$$
\int_{0}^{1}\left|\sum_{n \leq P / d} e\left(\alpha n^{3}\right)\right|^{8} d \alpha
$$

By Lemma $\mathrm{B}$ this is $\ll(P / d)^{5}$. It follows that

$$
\mathcal{J}(\mathfrak{A}) \ll P^{5} \sum_{d>(\log P)^{\varrho}} d^{-5 / 4} \ll P^{5}(\log P)^{-\varrho / 4} .
$$

We now turn our attention to $\mathcal{J}(\mathfrak{B})$. By assumption,

$$
\mathcal{J}(\mathfrak{B}) \ll P^{3 / 4}(\log P)^{\gamma} \int_{\mathfrak{m}}\left|\sum_{(x, y) \in \mathfrak{B}} e\left(\alpha\left(x^{3}-y^{3}\right)\right)\right| \prod_{j=4}^{8}\left|G_{j}(\alpha)\right| d \alpha .
$$

Upon replacing the range of integration by a unit interval and applying Hölder's inequality we find that $\mathcal{J}(\mathfrak{B})$ is

$$
\begin{aligned}
\ll P^{3 / 4}(\log P)^{\gamma}\left(\int_{0}^{1} \mid \sum_{(x, y) \in \mathfrak{B}} e\left(\alpha\left(x^{3}-y^{3}\right)\right)\right. & \left.\left.G_{4}(\alpha)\right|^{2} d \alpha\right)^{1 / 2} \\
& \times \prod_{j=5}^{8}\left(\int_{0}^{1}\left|G_{j}(\alpha)\right|^{8} d \alpha\right)^{1 / 8} .
\end{aligned}
$$

Replacing $G_{4}(\alpha)$ by $F(\alpha)$ above majorizes the number of solutions of the underlying equation. It follows from this and (3.1) that

$$
\mathcal{J}(\mathfrak{B}) \ll P^{13 / 4}(\log P)^{\gamma}\left(\int_{0}^{1}\left|\sum_{(x, y) \in \mathfrak{B}} e\left(\alpha\left(x^{3}-y^{3}\right)\right)\right|^{2}|F(\alpha)|^{2} d \alpha\right)^{1 / 2} .
$$

The integral in (3.4) is equal to the number of solutions of

$$
x_{1}^{3}-y_{1}^{3}+z_{1}^{3}=x_{2}^{3}-y_{2}^{3}+z_{2}^{3}
$$

with $x_{j} \in \mathcal{G}_{1}, y_{j} \in \mathcal{G}_{1}, 1 \leq z_{j} \leq P$, and $\left(x_{j}, y_{j}\right) \leq(\log P)^{\varrho}$ where both $x_{1}$ and $x_{2}$ have prime factors in the interval $\mathcal{Q}=\left((\log P)^{\varrho}, P^{1 / 7}\right]$. Thus $x_{j}$ may be written in the form $x_{j}=n_{j} m_{j}$ where $m_{j}$ is a prime in $\mathcal{Q}$ and $\left(m_{j}, y_{j}\right)=1$. Set

$$
f_{m}(\alpha)=\sum_{\substack{y=1 \\(y, m)=1}}^{P} e\left(\alpha y^{3}\right), \quad g_{m}(\alpha)=\sum_{1 \leq u \leq P / m} e\left(\alpha u^{3}\right) .
$$

Then

$$
\sum_{m_{1}, m_{2} \in \mathcal{Q}} \int_{0}^{1} f_{m_{1}}(-\alpha) g_{m_{1}}\left(\alpha m_{1}^{3}\right) f_{m_{2}}(\alpha) g_{m_{2}}\left(-\alpha m_{2}^{3}\right)|F(\alpha)|^{2} d \alpha
$$


is the number of solutions of

$$
u_{1}^{3} m_{1}^{3}-y_{1}^{3}+z_{1}^{3}=u_{2}^{3} m_{2}^{3}-y_{2}^{3}+z_{2}^{3}
$$

with $m_{j} \in \mathcal{Q}, 1 \leq y_{j} \leq P,\left(y_{j}, m_{j}\right)=1,1 \leq z_{j} \leq P$, and $1 \leq u_{j} \leq P / m_{j}$. This majorizes the quantity in (3.5). By the Cauchy-Schwarz inequality, the latter quantity is at most

$$
\begin{aligned}
\sum_{m_{1}, m_{2} \in \mathcal{Q}}\left(\int_{0}^{1} \mid f_{m_{1}}(\alpha)\right. & \left.\left.g_{m_{1}}\left(\alpha m_{1}^{3}\right) F(\alpha)\right|^{2} d \alpha\right)^{1 / 2} \\
& \times\left(\int_{0}^{1}\left|f_{m_{2}}(\alpha) g_{m_{2}}\left(\alpha m_{2}^{3}\right) F(\alpha)\right|^{2} d \alpha\right)^{1 / 2} \\
= & \left(\sum_{m \in \mathcal{Q}}\left(\int_{0}^{1}\left|f_{m}(\alpha) g_{m}\left(\alpha m^{3}\right) F(\alpha)\right|^{2} d \alpha\right)^{1 / 2}\right)^{2} .
\end{aligned}
$$

Combining this with (3.4) we deduce that

$$
\mathcal{J}(\mathfrak{B}) \ll P^{13 / 4}(\log P)^{\gamma} \sum_{m \in \mathcal{Q}}\left(\int_{0}^{1}\left|f_{m}(\alpha) g_{m}\left(\alpha m^{3}\right) F(\alpha)\right|^{2} d \alpha\right)^{1 / 2} .
$$

By the Cauchy-Schwarz inequality, the integral above is

$$
\ll\left(\int_{0}^{1}\left|f_{m}(\alpha)\right|^{2}|F(\alpha)|^{4} d \alpha\right)^{1 / 2}\left(\int_{0}^{1}\left|g_{m}\left(\alpha m^{3}\right)\right|^{4}\left|f_{m}(\alpha)\right|^{2} d \alpha\right)^{1 / 2} .
$$

By considering the underlying diophantine equation, the first integral in (3.6) is bounded by

$$
\int_{0}^{1}|F(\alpha)|^{6} d \alpha \leq\left(\int_{0}^{1}|F(\alpha)|^{4} d \alpha\right)^{1 / 2}\left(\int_{0}^{1}|F(\alpha)|^{8} d \alpha\right)^{1 / 2} .
$$

Using Lemmata A and B, we conclude that

$$
\mathcal{J}(\mathfrak{B}) \ll P^{33 / 8}(\log P)^{\gamma} \sum_{m \in \mathcal{Q}}\left(\int_{0}^{1}\left|g_{m}\left(\alpha m^{3}\right)\right|^{4}\left|f_{m}(\alpha)\right|^{2} d \alpha\right)^{1 / 4} .
$$

Set $M_{0}=(\log P)^{\varrho}$ and, for $t>0, M_{t}=2 M_{t-1}$. We decompose $\mathcal{Q}$ into dyadic blocks $\mathcal{M}_{t}=\left(M_{t-1}, M_{t}\right]$. By Hölder's inequality,

$$
\begin{gathered}
\sum_{m \in \mathcal{M}_{t}}\left(\int_{0}^{1}\left|g_{m}\left(\alpha m^{3}\right)\right|^{4}\left|f_{m}(\alpha)\right|^{2} d \alpha\right)^{1 / 4} \\
\leq M_{t-1}^{3 / 4}\left(\sum_{m \in \mathcal{M}_{t}} \int_{0}^{1}\left|g_{m}\left(\alpha m^{3}\right)\right|^{4}\left|f_{m}(\alpha)\right|^{2} d \alpha\right)^{1 / 4} .
\end{gathered}
$$


The sum on the right-hand side of (3.8) is equal to the number of solutions of

$$
m^{3}\left(u_{1}^{3}+u_{2}^{3}\right)+y_{1}^{3}=m^{3}\left(u_{3}^{3}+u_{4}^{3}\right)+y_{2}^{3}
$$

with $1 \leq u_{j} \leq P / m,\left(y_{j}, m\right)=1,1 \leq y_{j} \leq P$, and $m \in \mathcal{M}_{t}$. Since $u_{j} \leq P / m<P / M_{t-1}$, it follows from Lemma $\mathrm{C}$ combined with (3.7) and (3.8) that

$$
\begin{aligned}
\mathcal{J}(\mathfrak{B}) & \ll P^{33 / 8}(\log P)^{\gamma} \sum_{t} M_{t-1}^{3 / 4}\left(P^{7 / 2} M_{t-1}^{-9 / 2}(\log P)^{5}\right)^{1 / 4} \\
& \ll P^{5}(\log P)^{\gamma+5 / 4-3 \varrho / 8} .
\end{aligned}
$$

Upon interchanging the rôles of $x$ and $y$, it is clear that $\mathcal{J}(\mathfrak{C})$ may be treated in a manner identical to that of $\mathcal{J}(\mathfrak{B})$-leading to the bound

$$
\mathcal{J}(\mathfrak{C}) \ll P^{5}(\log P)^{\gamma+5 / 4-3 \varrho / 8} .
$$

We now consider the final piece, $\mathcal{J}(\mathfrak{D})$. We first observe that

$$
\begin{aligned}
\mathcal{J}(\mathfrak{D})= & \int_{\mathfrak{m}}\left|\widehat{G}_{1}(\alpha)\right|^{2} \prod_{j=3}^{8}\left|G_{j}(\alpha)\right| d \alpha \\
& -\sum_{d>(\log P) \varrho} \int_{\mathfrak{m}} \sum_{\substack{x, y \in \mathcal{X} \cap \mathcal{G}_{1} \\
(x, y)=d}} e\left(\alpha\left(x^{3}-y^{3}\right)\right) \prod_{j=3}^{8}\left|G_{j}(\alpha)\right| d \alpha .
\end{aligned}
$$

After extending the range of integration, the second integral in (3.11), by Hölder's inequality, has absolute value at most

$$
\left(\int_{0}^{1}\left|\sum_{\substack{x, y \in \mathcal{X} \cap \mathcal{G}_{1} \\(x, y)=d}} e\left(\alpha\left(x^{3}-y^{3}\right)\right)\right|^{4} d \alpha\right)^{1 / 4} \prod_{j=3}^{8}\left(\int_{0}^{1}\left|G_{j}(\alpha)\right|^{8} d \alpha\right)^{1 / 8}
$$

and the first integral in (3.12) is equal to the number of solutions of

$$
x_{1}^{3}-y_{1}^{3}+x_{2}^{3}-y_{2}^{3}=x_{3}^{3}-y_{3}^{3}+x_{4}^{3}-y_{4}^{3}
$$

with $x_{i}$ and $y_{i}$ in $\mathcal{X} \cap \mathcal{G}_{1}$ and $\left(x_{j}, y_{j}\right)=d$. This is no more than the number of solutions of the same equation with $x_{i}$ and $y_{i}$ at most $P / d$. It follows from these observations and (3.1) that the second integral in (3.11) has absolute value at most

$$
P^{15 / 4}\left(\int_{0}^{1}\left|\sum_{n \leq P / d} e\left(\alpha n^{3}\right)\right|^{8} d \alpha\right)^{1 / 4} .
$$

By Lemma B this is $\ll P^{5} d^{-5 / 4}$. Summing over $d$ we conclude that

$$
\mathcal{J}(\mathfrak{D})=\int_{\mathfrak{m}}\left|\widehat{G}_{1}(\alpha)\right|^{2} \prod_{j=3}^{8}\left|G_{j}(\alpha)\right| d \alpha+O\left(P^{5}(\log P)^{-\varrho / 4}\right) .
$$


Combining this with (3.2), (3.3), (3.9), and (3.10), Lemma D is proved upon taking

$$
\varrho \geq \max \left(8 \delta, \frac{1}{3}(16 \delta+10+8 \gamma)\right) .
$$

4. General treatments. In order to prove the Theorem we need the following bound.

Lemma E (Vaughan, [7, §2]). Let

$$
\widehat{F}(\alpha)=\sum_{n \in \mathcal{X}} e\left(\alpha n^{3}\right) .
$$

Then

$$
\int_{0}^{1}|\widehat{F}(\alpha)|^{8} d \alpha \ll P^{5}(\log P)^{\varepsilon-3} .
$$

(Vaughan [7] gives a more general treatment for $k$ th powers.)

Proof of the Theorem. Put

$$
I=\int_{\mathfrak{m}} \prod_{i=1}^{8}\left|G_{i}(\alpha)\right| d \alpha .
$$

By Hölder's inequality,

$$
I \leq \prod_{j=2}^{8}\left(\int_{\mathfrak{m}}\left|G_{1}(\alpha)\right|\left|G_{j}(\alpha)\right|^{7} d \alpha\right)^{1 / 7} .
$$

It suffices to treat

$$
I_{j}=\int_{\mathfrak{m}}\left|G_{1}(\alpha)\right|\left|G_{j}(\alpha)\right|^{7} d \alpha .
$$

By Lemma D, for any $\delta$,

$$
I_{j} \ll \int_{\mathfrak{m}}\left|\widehat{G}_{j}(\alpha)\right|^{2}\left|G_{1}(\alpha)\right|\left|G_{j}(\alpha)\right|^{5} d \alpha+P^{5}(\log P)^{-\delta} .
$$

By Hölder's inequality,

$$
\begin{aligned}
I_{j} \ll & \left(\int_{\mathfrak{m}}\left|\widehat{G}_{j}(\alpha)\right|^{7}\left|G_{1}(\alpha)\right| d \alpha\right)^{2 / 7}\left(\int_{\mathfrak{m}}\left|G_{j}(\alpha)\right|^{7}\left|G_{1}(\alpha)\right| d \alpha\right)^{5 / 7} \\
& +P^{5}(\log P)^{-\delta} \\
= & I_{j}^{5 / 7}\left(\int_{\mathfrak{m}}\left|\widehat{G}_{j}(\alpha)\right|^{7}\left|G_{1}(\alpha)\right| d \alpha\right)^{2 / 7}+P^{5}(\log P)^{-\delta}
\end{aligned}
$$

whence

$$
I_{j} \ll \int_{\mathfrak{m}}\left|\widehat{G}_{j}(\alpha)\right|^{7}\left|G_{1}(\alpha)\right| d \alpha+P^{5}(\log P)^{-\delta}
$$


(where we have altered the value of $\delta$ without apology). Applying Hölder's inequality again,

$$
I_{j} \ll\left(\int_{\mathfrak{m}}\left|\widehat{G}_{j}(\alpha)\right|^{8} d \alpha\right)^{7 / 8}\left(\int_{\mathfrak{m}}\left|G_{1}(\alpha)\right|^{8} d \alpha\right)^{1 / 8}+P^{5}(\log P)^{-\delta} .
$$

Extending the range of integration on the first integral we obtain

$$
\int_{\mathfrak{m}}\left|\widehat{G}_{j}(\alpha)\right|^{8} d \alpha \leq \int_{0}^{1}\left|\widehat{G}_{j}(\alpha)\right|^{8} d \alpha .
$$

As $\mathcal{G}_{j} \cap \mathcal{X} \subseteq \mathcal{X}$, we find that, upon comparing the underlying diophantine equations,

$$
\int_{0}^{1}\left|\widehat{G}_{j}(\alpha)\right|^{8} d \alpha \leq \int_{0}^{1}|\widehat{F}(\alpha)|^{8} d \alpha .
$$

It follows from Lemma $\mathrm{E}$ that

$$
I_{j} \ll P^{35 / 8}(\log P)^{-21 / 8+\varepsilon}\left(\int_{\mathfrak{m}}\left|G_{1}(\alpha)\right|^{8} d \alpha\right)^{1 / 8}+P^{5}(\log P)^{-\delta} .
$$

To treat the integral in (4.3), we employ Lemma D to find that

$$
I_{1}=\int_{\mathfrak{m}}\left|G_{1}(\alpha)\right|^{8} d \alpha \ll \int_{\mathfrak{m}}\left|\widehat{G}_{1}(\alpha)\right|^{2}\left|G_{1}(\alpha)\right|^{6} d \alpha+P^{5}(\log P)^{-\delta} .
$$

By Hölder's inequality we conclude that

$$
I_{1} \ll I_{1}^{3 / 4}\left(\int_{\mathfrak{m}}\left|\widehat{G}_{1}(\alpha)\right|^{8} d \alpha\right)^{1 / 4}+P^{5}(\log P)^{-\delta},
$$

whence, for a different $\delta$,

$$
I_{1} \ll \int_{\mathfrak{m}}\left|\widehat{G}_{1}(\alpha)\right|^{8} d \alpha+P^{5}(\log P)^{-\delta} .
$$

Using the treatment already provided, we have, from Lemma E,

$$
I_{1} \ll P^{5}(\log P)^{\varepsilon-3} .
$$

Placing this bound in (4.3) and (4.1), the Theorem is proved.

The quantity bounded in Lemma $\mathrm{E}$,

$$
\int_{0}^{1}|\widehat{F}(\alpha)|^{8} d \alpha
$$

is equal to

$$
J=\#\left\{x_{i} \in \mathcal{X}: x_{1}^{3}+x_{2}^{3}+x_{3}^{3}+x_{4}^{3}=x_{5}^{3}+x_{6}^{3}+x_{7}^{3}+x_{8}^{3}\right\} .
$$


By utilizing sieve techniques (see [1, Theorem 3.4]),

$$
\# \mathcal{X} \asymp \frac{P \log \log P}{\log P} .
$$

We therefore expect that $J \ll P^{5}(\log P)^{\varepsilon-8}$. This is likely to be very difficult. The author gladly offers a pony for a proof of this assertion.

The proof of the Theorem is especially wasteful if the cardinality of $\mathcal{G}_{i} \cap \mathcal{X}$ is appreciably smaller than that of $\mathcal{X}$. If this is the case, an amended form of Lemma E may provide more desirable results when implemented in (4.2).

Before we apply the Theorem, we need a suitable minor arc estimate. The following result of Vaughan is sufficient for the applications that we shall consider.

Lemma F (Vaughan, [6, Lemma 1]). Let

$$
\mathfrak{m}=\left\{\alpha:\left|\alpha-\frac{a}{q}\right| \leq \frac{1}{q P^{9 / 4}} \text { with }(a, q)=1 \text { implies } q>P^{3 / 4}\right\} .
$$

Then, uniformly for $\alpha \in \mathfrak{m}$,

$$
F(\alpha) \ll P^{3 / 4}(\log P)^{1 / \pi+\varepsilon} .
$$

The exponent $1 / \pi$ in Lemma $\mathrm{F}$ may be reduced to $1 / 4$ by employing bounds of Hall and Tenenbaum (see [2]) in the argument of Vaughan.

5. Proof of Corollary 1. Our choice for the generating functions $G_{i}(\alpha)$ in the Theorem is to set them all equal to $F(\alpha)$. The minor arc integral corresponds to $r_{8,3}(n)$, the number of representations of $n$ as the sum of eight positive cubes. As usual, let $P=\left[n^{1 / 3}\right]$. By Dirichlet's theorem, for any real $\alpha$ we can find integers $a$ and $q$ with $(a, q)=1$ and $1 \leq q \leq P^{9 / 4}$ where

$$
\left|\alpha-\frac{a}{q}\right| \leq \frac{1}{q P^{9 / 4}}
$$

Set

$$
\mathfrak{M}(a, q)=\left\{\alpha:\left|\alpha-\frac{a}{q}\right| \leq \frac{1}{q P^{9 / 4}}\right\}, \quad \mathfrak{M}=\bigcup_{a, q}^{*} \mathfrak{M}(a, q)
$$

where the $*$ signifies that the union is taken over all $1 \leq a \leq q \leq P^{3 / 4}$ with $(a, q)=1$. Then $\mathfrak{M} \subset \mathcal{V}$ where

$$
\mathcal{V}=\left[\frac{1}{P^{9 / 4}}, 1+\frac{1}{P^{9 / 4}}\right] \text {. }
$$

It shall be convenient to work on $\mathcal{V}$ and not the unit interval $[0,1]$. Let $\mathfrak{m}=\mathcal{V} \backslash \mathfrak{M}$. We may treat the major arcs $\mathfrak{M}$ using standard techniques (see, 
for example, [5, Chapter 2]) to obtain

$$
\int_{\mathfrak{M}} F(\alpha)^{8} e(-n \alpha) d \alpha=\mathfrak{S}_{8,3}(n) \frac{\Gamma(4 / 3)^{8}}{\Gamma(8 / 3)} n^{5 / 3}+O\left(n^{5 / 3-\delta}\right)
$$

for some explicit positive $\delta$. By construction, every $\alpha \in \mathfrak{m}$ satisfies the conditions of Lemma F so, by the Theorem,

$$
\int_{\mathfrak{m}} F(\alpha)^{8} e(-n \alpha) d \alpha \leq \int_{\mathfrak{m}}|F(\alpha)|^{8} d \alpha \ll P^{5}(\log P)^{\varepsilon-3} .
$$

This completes the proof of the corollary.

6. Proof of Corollary 2. Let

$$
G(\alpha)=\sum_{n \in \mathcal{P}} e\left(\alpha n^{3}\right)
$$

where $\mathcal{P}$ denotes the set of primes at most $P=\left[n^{1 / 3}\right]$. Let $\mathfrak{M}$ and $\mathfrak{m}$ be as defined in the proof of Corollary 1 . Set $W=(\log P)^{12}$ and

$$
\mathfrak{N}(a, q)=\left\{\alpha \in \mathcal{V}:\left|\alpha-\frac{a}{q}\right| \leq \frac{W}{q P^{3}}\right\}, \quad \mathfrak{N}=\bigcup_{a, q}^{*} \mathfrak{N}(a, q)
$$

where the $*$ signifies the union is taken over all $1 \leq a \leq q \leq W$ with $(a, q)=1$. The $\mathfrak{N}(a, q)$ are disjoint and $\mathfrak{N} \subseteq \mathfrak{M}$. Let $\mathfrak{n}=\mathfrak{M} \backslash \mathfrak{N}$, the pruned sections of the major $\operatorname{arcs} \mathfrak{N}$. Then

$$
\begin{aligned}
R_{6,2}(n) & =\int_{0}^{1} G(\alpha)^{2} F(\alpha)^{6} e(-n \alpha) d \alpha \\
& =\left(\int_{\mathfrak{N}}+\int_{\mathfrak{m}}+\int_{\mathfrak{n}}\right) G(\alpha)^{2} F(\alpha)^{6} e(-n \alpha) d \alpha .
\end{aligned}
$$

By the Theorem,

$$
\int_{\mathfrak{m}}|G(\alpha)|^{2}|F(\alpha)|^{6} d \alpha \ll P^{5}(\log P)^{\varepsilon-3} .
$$

By Hölder's inequality,

$$
\int_{\mathfrak{n}}|G(\alpha)|^{2}|F(\alpha)|^{6} d \alpha \leq\left(\int_{\mathfrak{n}}|G(\alpha)|^{8} d \alpha\right)^{1 / 4}\left(\int_{\mathfrak{n}}|F(\alpha)|^{8} d \alpha\right)^{3 / 4} .
$$

Extending the range on the first integral to a unit interval and subsequently considering the underlying diophantine equation, we find the first integral is, by Lemma B, $O\left(P^{5}\right)$. In order to treat the remaining integral over $\mathfrak{n}$ we 
employ a result of Vaughan [8, Lemma 5.1] which provides that

$$
\int_{\mathfrak{n}}|F(\alpha)|^{8} d \alpha \ll P^{5} W^{\varepsilon-1 / 3} .
$$

Thus

$$
\int_{\mathfrak{n}}|G(\alpha)|^{2}|F(\alpha)|^{6} d \alpha \ll P^{5}(\log P)^{\varepsilon-3},
$$

whence, from (6.1) and (6.2),

$$
R_{6,2}(n)=\int_{\mathfrak{N}} G(\alpha)^{2} F(\alpha)^{6} e(-n \alpha) d \alpha+O\left(n^{5 / 3}(\log n)^{\varepsilon-3}\right) .
$$

As usual, we implement auxiliary functions to approximate $G(\alpha)$ and $F(\alpha)$ to obtain the main term on the major arcs $\mathfrak{N}$. Let

$$
v^{*}(\beta)=\sum_{2 \leq u \leq P^{3}} u^{-2 / 3}(\log u)^{-1} e(\beta u), \quad v(\beta)=\frac{1}{3} \sum_{1 \leq u \leq P^{3}} u^{-2 / 3} e(\beta u),
$$

and $S(q, a)$ and $S^{*}(q, a)$ be as defined in the statement of the corollary. Set

$$
\begin{aligned}
V^{*}(\alpha, q, a) & =\phi(q)^{-1} S^{*}(q, a) v^{*}\left(\alpha-\frac{a}{q}\right), \\
V(\alpha, q, a) & =q^{-1} S(q, a) v\left(\alpha-\frac{a}{q}\right) .
\end{aligned}
$$

By standard estimates [5, Theorem 4.1],

$$
F(\alpha)-V(\alpha, q, a) \ll q^{1 / 2+\varepsilon},
$$

from which it follows that (see [5, Chapter 4])

$$
\int_{\mathfrak{N}} G(\alpha)^{2} F(\alpha)^{6} e(-n \alpha) d \alpha
$$

is equal to

$$
\sum_{q=1}^{W} \sum_{\substack{a=0 \\(a, q)=1}}^{q} \int_{\mathfrak{N}(a, q)} G(\alpha)^{2} V(\alpha, q, a)^{6} e(-n \alpha) d \alpha+O\left(n^{5 / 3-\delta}\right)
$$

for some explicit $\delta>0$. Following Roth [4, Lemma 2], for $\alpha \in \mathfrak{N}(a, q)$,

$$
G(\alpha)-V^{*}(\alpha, q, a) \ll P e^{-c_{1} \sqrt{\log P}}
$$

for some positive constant $c_{1}$ (a result which rests upon the Theorem of Siegel-Walfisz). It follows that for $\alpha \in \mathfrak{N}(a, q)$,

$$
G(\alpha)^{2}-V^{*}(\alpha, q, a)^{2} \ll P e^{-c_{1} \sqrt{\log P}}\left(P e^{-c_{1} \sqrt{\log P}}+V^{*}(\alpha, q, a)\right) .
$$


From (6.3) and (6.4) we conclude that

$$
\begin{array}{r}
R_{6,2}(n)=\sum_{q=1}^{W} \sum_{\substack{a=1 \\
(a, q)=1}}^{q} \int_{\mathfrak{N}(a, q)} V^{*}(\alpha, q, a)^{2} V(\alpha, q, a)^{6} e(-n \alpha) d \alpha \\
+O\left(K(n)+n^{5 / 3}(\log n)^{\varepsilon-3}\right)
\end{array}
$$

where

$$
\begin{aligned}
K(n)=\sum_{q=1}^{W} \sum_{\substack{a=0 \\
(a, q)=1}} \int_{\mathfrak{N}(a, q)} P e^{-c_{1} \sqrt{\log P}} \\
\quad \times\left(P e^{-c_{1} \sqrt{\log P}}+\left|V^{*}(\alpha, q, a)\right|\right)|V(\alpha, q, a)|^{6} d \alpha .
\end{aligned}
$$

By a direct calculation, $K(n) \ll n^{5 / 3}(\log n)^{-\varrho}$ for every $\varrho>0$. It follows that

$$
\begin{aligned}
R_{6,2}(n)= & \sum_{q=1}^{W} \sum_{\substack{a=1 \\
(a, q)=1}}^{q} q^{-6} \phi(q)^{-2} S(q, a)^{6} S^{*}(q, a)^{2} \\
& \times e\left(-\frac{a n}{q}\right) \int_{-W / q P^{3}}^{W / q P^{3}} v^{*}(\beta)^{2} v(\beta)^{6} e(-n \beta) d \beta+O\left(n^{5 / 3}(\log n)^{\varepsilon-3}\right)
\end{aligned}
$$

We complete the proof of Corollary 2 by appealing to classical techniques (see [5, Chapter 2]). We first extend the sum on $q$ to infinity and the range of integration to infinity (with acceptable error). The result then follows standard estimates for the function $v(\beta)$ and straightforward calculations involving $v^{*}(\beta)$.

This proof of Corollary 2 was benefitted by a conversation with T. D. Wooley.

It may be easily deduced that $\mathfrak{S}(n)$, the singular series, satisfies $\mathfrak{S}(n) \gg$ 1 (see [5, Chapter 2]). As experts in the field will realize, a delicate treatment of the associated singular series for the representation of a number as the sum of three cubes and the cube of a prime leads to a mean-square result providing that the expected asymptotic formula for the representation as the sum of three cubes and the cube of a prime holds almost always (in the sense of natural density).

\section{References}

[1] H. Halberstam and H.-E. Richert, Sieve Methods, Academic Press, London, 1974.

[2] R. Hall and G. Tenenbaum, Divisors, Cambridge Univ. Press, London, 1988. 
[3] C. Hooley, On the numbers that are representable as the sum of two cubes, J. Reine Angew. Math. 314 (1980), 146-173.

[4] K. F. Roth, On Waring's problem for cubes, Proc. London Math. Soc. (2) 53 (1951), 268-279.

[5] R. C. Vaughan, The Hardy-Littlewood Method, Cambridge Univ. Press, London, 1981.

[6] -, On Waring's problem for cubes, J. Reine Angew. Math. 365 (1986), 122-170.

[7] —, On Waring's problem for smaller exponents, II, Mathematika 33 (1986), 6-22.

[8] -, A new iterative method in Waring's problem, Acta Math. 162 (1989), 1-71.

DEPARTMENT OF MATHEMATICS

VANDERBILT UNIVERSITY

1326 STEVENSON CENTER

NASHVILLE, TENNESSEE 37240-0001

U.S.A.

E-mail: BOKLAN@ATHENA.CAS.VANDERBILT.EDU

Received on 15.12.1992

and in revised form on 9.4.1993 